\title{
MULTI-STATE MODELS OF HIV/AIDS BY HOMOGENEOUS SEMI-MARKOV PROCESS
}

\author{
Zelalem Getahun Dessie \\ Department of Statistics, College of Science, Bahir Dar University, Bahir Dar, Ethiopia
}

Received 2014-07-16; Revised 2014-07-21; Accepted 2014-08-04

\begin{abstract}
Multi-state stochastic models are useful tools for studying complex dynamics such as chronic diseases. The purpose of this study is to determine factors associated with the progression between different stages of the disease and to model the progression of HIV/AIDS disease of an individual patient under ART follow-up using semi-Markov processes. A sample of 1456 patients has been taken from a hospital record at Amhara Referral Hospitals, Amhara Region, Ethiopia, who have been under ART follow up from June 2006 to August 2013. The states of disease progression adopted in the multi-state model were defined based on of the following CD4 cell counts: $\geq 500$ (SI); 200 to 499 (SII); $<200$ (SIII); and Death (D). The first three states are named as good. Female patients were 1.6 times more likely to move from state 2 to state 1 than those of male patients (adjusted HR $=1.60, \mathrm{CI}=1.02-2.49$ ). Patients, who is not drug addicted, were 2.49 times more likely to move from state 3 to state 2 than those of drug addicted(adjusted HR = $2.67, \mathrm{CI}=1.52-4.68)$. Patients with tuberculosis were 2.67 times more likely to move from state 3 to state 4 than those with no tuberculosis (adjusted HR $=2.67, \mathrm{CI}=1.52-4.68$ ). On the other hand, the probability of staying in same state until a given number of month decreases with increasing time. Multi-state modeling is a powerful approach for studying chronic diseases and estimating factors associated with transitions between each stage of progression. The major predictors of the intensity of transitions between different states of HIV/AIDS patients were gender, age, drug addicted and TB status. The dynamic nature of the AIDS progression is confirmed with particular findings that there is more likely to be in worse state than better one unless interventions are made.
\end{abstract}

Keywords: Disease Progression, Markov Chain, HIV/AIDS

\section{INTRODUCTION}

The rate of spread of the HIV/AIDS epidemic has reached a shocking level. The expansion of the epidemic has now become a burning issue globally and this is particularly so more important in developing countries. The disease being one without any cure is still account for economic, social and health crises in many developing countries. On a global scale, the HIV epidemic has stabilized, although with unacceptably high levels of new HIV infections and AIDS deaths. An estimated 34 million people worldwide were living with HIV in 2011 among which 23.5 million are living in subSaharan Africa. Ethiopia is one of the countries hardest hit by HIV/AIDS epidemic. An estimated of 790,000 were living with HIV in 2011(UNAIDS, 2013).

Most studies of AIDS have analyzed the factors associated with therapeutic failure, such as death, opportunistic disease, detect Viral Load (VL) or low levels of immunity, using semi parametric Cox models (Pradeep et al., 2010; Muralidhar et al., 2010; Keiser et al., 2010; Koethe et al., 2010; Neuhaus et al., 2010). However, it is also important to consider chronicity and the relatively slow progression of the disease and to assess the multiple immune states resulting from the effect of the virus on the human immune system. The use of multi-state Markov models to analyze the factors associated with transitions between different states of 
chronicity has been suggested for chronic diseases and the cost-effectiveness of various therapeutic regimes (Shih et al., 2007; Pan et al., 2007; Gil et al., 2007).

Recent studies have shown that the predicted probability of patients that changing their status given his/her current status allows the measurements of medical scientific progresses due to the advances in the treatment of the HIV/AIDS (D'Amico et al., 2009). Since the discovery of HIV/AIDS, numerous mathematical models Blasi and Manca (2004) have been developed to describe infectious disease transmission dynamics, understand the mechanisms of epidemic propagation, assess the impact of interventions on public health or forecast the future of epidemics. Among recent papers in biomedicine, Masala et al. (2014; Goshu and Dessie, 2013; Giuseppe et al., 2007) analyzed HIV/AIDS dynamic evolution as defined by CD4 levels from a macroscopic point of view by means of homogeneous semi-Markov stochastic processes. Numerical analyses of the homogeneous semi-Markov process are dealt by Corradi et al. (2004; Janssen and Manca, 2001). Other more readings include (Davidov and Zelen, 2000; Viladent and Van Ackere, 2007; Satten and Sternberg, 1999; Baryarama et al., 2005).

In this study, the author proposes homogeneous Markov process to study the evolution of HIV/AIDS. The homogeneous approach considered is a piecewise Markov process, with the transition intensity functions being step functions. The author is interested in determining factors associated with the progression between different stages of the disease. Several researchers have studied Markov processes with covariates and a procedure to obtain the parameters in a model with covariates has been reported (Maciulis et al., 2009; Gentlemann et al., 1985; Mathieu et al., 2007; Pèrez-Ocón et al., 2001). The author also present the results of modelling of the waiting time of HIV/AIDS so as to predict the future waiting time of a patient. This is: The conditional probability of staying in the starting state until month $\mathrm{t}$.

\section{MATERIALS AND METHODS}

\subsection{Ethics Statement}

This investigation was conducted according to the principles expressed in the Declaration of Bahir Dar University, Ethiopia. It was approved by the research ethics committee at the University of Bahir Dar and all participants who agreed to participate in this study signed a consent form.

\subsection{Data and Model Descriptions}

The target population for this study was patients under the follow up of ART at Amhara Referral Hospitals, Amhara Region, Ethiopia from June 2006 to August 2013. Multistage sampling was used to select study subjects. First, all the Referral Hospitals in the Amhara Region were stratified in to Western and Eastern area. The calculated sample size was proportionally allocated to Eastern $(\mathrm{n}=482)$ and Western $(n=874)$ areas respectively. The sampling frame consists of $65000 \mathrm{HIV} / \mathrm{AIDS}$ patients who have visited the hospitals since the initiation of ART. The study may consider all HIV infected patients under ART whose age is $\geq 15$ years regardless of their treatment category during the study period in Amhara Referral Hospitals, Amhara Region, Ethiopia.

The states of disease progression adopted in the multi-state model were defined based on the following CD4 cell counts as in Oliveira et al. (2013; Giuseppe et al., 2007): $\geq 500$ cells $/ \mathrm{mm}^{3}(\mathrm{SI}) ; 350$ to 499 cells $/ \mathrm{mm}^{3}(\mathrm{SII})$; $<350$ cells $/ \mathrm{mm}^{3}$ (SIII); and death(D). The death state is considered to be an absorbing state-meaning that once a patient is in the death state she/he will never be in the others states and rather stays there forever. The time elapse between state transitions was determined using the difference (in months) between the dates of CD4 tests.

In Fig. 1 the graph model is pictured. It shows all the immunological states a HIV infected patient can go into. All the states apart from than Death are interrelated and also improvements are considered. It is also possible that an examination will show that the patient's state has not changed.

\subsection{Modelling Semi-Markov Processes}

\subsubsection{The Transition-Specific Semi-Markov Model}

A semi-Markov process is a stochastic process, $\{\mathrm{X}(\mathrm{t})$ : $t \geq 0$, where an embedded Markov chain governs the state to state transitions of the process while a separate probabilistic mechanism determines the time spent in each state. It is assumed that the transition probabilities depend on the current state and the time spent in each state depends upon the current and next state. Several authors have discussed the use of Markov processes techniques in health. The author cites only some of them, i.e., (Jackson et al., 2003; D'Amico et al., 2005; Foucher et al., 2005; Barbu and Limnios, 2009). 


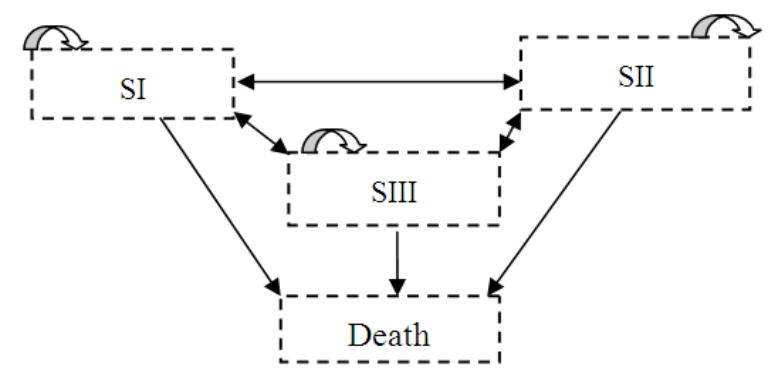

Fig. 1. Communication between the states of the process

In this part, Homogeneous Semi-Markov model are described by using the notation of Foucher et al. (2005).

On a complete probability space the author define two random variables. $X_{n}: \Omega \rightarrow S$ be the stochastic process with state space $S=\left\{S_{1}, S_{2} \ldots S_{m}\right\}$ and $T_{n}: \Omega \rightarrow \Re$ be the time of the $n^{\text {th }}$ transition, with $\Omega$ domain of the process and $\mathfrak{R}$ set real numbers.

The kernel $Q=\left[Q_{i j}\right]$ associated with the process and the transition matrix $P_{i j}$ of the embedded Markov chain is defined as follows Equation 1 and 2:

$$
\begin{aligned}
& Q_{i j}(t)=P\left[X_{n+1}=j, T_{n+1}-T_{n} \leq t \mid X_{n}=i\right] \\
& P_{i j}=\lim _{t \rightarrow \infty} Q_{i j}(t)
\end{aligned}
$$

And it is necessary to introduce the probability that the process may leave state $i$ in a time $t$ as Equation 3:

$$
H_{i}(t)=P\left[T_{n+1}-T_{n} \leq t \mid X_{n}=i\right]=\sum_{j=1}^{m} Q_{i j}(t)
$$

Foucher et al. (2005) defines the density probability function, of the waiting time in state $i$ before passing to state $j$ as follow Equation 4:

$$
f_{i j}\left(x, \theta_{i j}\right)=\lim _{h \rightarrow 0^{+}} \frac{P\left(x<T_{n+1}-T_{n}<x+h \mid X_{n+1}=j, X_{n}=i\right)}{h}
$$

where, $\theta_{i j}$ is the parameter vector of the density probability function $f_{i j}($,$) . The distribution and the value$ of parameters can vary between transitions. As usual in survival analysis, we deduce from $f_{i j}(\mathrm{x})$ the corresponding survival function and hazard function, respectively $S_{i j}(\mathrm{x})$ and $g_{i j}(\mathrm{x})$ Equation 5:

$$
\begin{array}{r}
P\left(x<T_{n+1}-T_{n}<x+h \mid T_{n+1}\right. \\
G_{i j}(x)=\lim _{h \rightarrow 0^{+}} \frac{\left.-T_{n} \geq x, X_{n+1}=j, X_{n}=i\right)}{h}
\end{array}
$$

The hazard function of the semi-Markovian process corresponds to the probability of jumping towards state $j$, given that the process occupies state $i$ for duration $x$ Equation 6:

$G_{i j}(x)=\frac{P_{i j} f_{i j}(x)}{S_{i .}(x)}$ with $\left\{\begin{array}{l}i \neq j \\ i, j \in E \\ \lambda_{i i}(x)=-\sum_{j=i} \lambda_{i j}(x)\end{array}\right.$

It is assumed that process spends some time in a given state and random time has distribution $G_{i j}(x)$, Weibull distribution: -The hazard function is defined as Equation 7:

$G_{i j}(x)=\theta_{i j}\left(\frac{1}{\sigma_{i j}}\right)^{\theta_{i j}} x^{\theta_{i j}-1}, x \geq 0$

Foucher et al. (2005) also defines the hazard function with covariates as follow:$G_{i j}\left(x, z_{i j}\right)=G_{0, i j}(x) \exp \left(\beta_{i j}^{T} z_{i j}\right)$, where $z_{i j}=\left(z_{i j}^{1}, z_{i j}^{2}, \ldots, z_{i j}^{n_{i j}}\right)$ the vector of $n_{i j}$ covariates, specific to the transition $i \rightarrow j$. In the same paper, they proved that the Parameter estimations and likelihood methods Equation 8:

$$
\begin{aligned}
& L=\prod_{h} \prod_{r=1}^{m}\left\{P_{X_{r-1}^{h}, X_{r}^{h}} f_{X_{r-1}^{h}, X_{r}^{h}}\left(T_{h, r}-T_{h, r-1, Z_{X_{r-1}^{h}}, X_{r}^{h}}\right)\right\}^{\delta_{h, r}} \\
& \left\{S_{X_{r-1}^{h} \cdot}\left(T_{h, r}-T_{\left.h, r-1, Z_{X_{r-1}^{h}}, X_{r}^{h}\right)}\right)\right\}^{1-\delta_{h, r}}
\end{aligned}
$$

where, $\delta_{h, r}$ is equal to 1 if the transition $\mathrm{r}$ is observed for the individual $\mathrm{h}$ and 0 if censored.

\subsubsection{Semi-Markov for Predicting the Probability of Waiting Time}

After solving the evolution equations of the semiMarkov model, it is appropriate to give some concrete applications of these processes as models of evolution of the probability of waiting time of some system.

In this part, Numerical solution of the Homogeneous Semi-Markov model is described by using the notation of Giuseppe et al. (2007).

For any homogeneous semi-Markov process $\{X(t), t \geq 0\}$, the transition probabilities are given by (9) for which the solutions should be obtained using the progression (10):

$\varphi_{i j}(t)=P[X(t)=j \mid X(0)=i]$ 


$$
\varphi_{i j}(t)=\left(1-H_{i}(t)\right) \delta_{i j}+\sum_{l=1}^{m} \int_{0}^{t} Q_{i l}(\tau) \varphi_{l j}(t-\tau) d \tau
$$

Here $\delta_{i j}$ represents the Kronecker delta. An approximate solution of (10) can be obtained using the general numerical integration formula given in Corradi et al. (2004). In the same paper, they proved that the numerical solution of the process converges to the discrete time HSMP described as an infinite countable linear system Equation 11:

$$
\varphi_{i j}^{h}(k h)=d_{i j}^{h}(k h)+\sum_{l=1}^{m} \sum_{\tau=1}^{k} v_{i j}^{h}(\tau h) \varphi_{i j}^{h}((k-\tau) h)
$$

where, $h$ stands for the step measure of the approximation and:

$$
\begin{aligned}
& d_{i j}^{h}(k h)= \begin{cases}0 & \text { if } i \neq j \\
1-H_{i}^{h}(k h) & \text { if } i=j\end{cases} \\
& v_{i j}^{h}(k h)= \begin{cases}0 & \text { if } k \neq 0 \\
Q_{i j}^{h}(k h)-Q_{i j}^{h}((k-1) h) & \text { if } i=j\end{cases}
\end{aligned}
$$

In matrix form, Equation 12 becomes:

$$
\Phi^{h}(k h)-\sum_{\tau=1}^{k} V(\tau h) \Phi^{h}((k-\tau) h)=D^{h}(k h)
$$

The fact that the matrix $\Phi^{h}(k h)$ is stochastic is already proved in Corradi et al. (2004; Janssen and Manca, 2001). For solving the evolution Equation 13, Corradi et al. (2004) proposed the following algorithm with suggested matrix form Equation 14:

$$
V^{T} \Phi^{T}=D^{T}
$$

The variables involved are the following:

$$
\begin{aligned}
m= & \text { number of states of SMP } \\
T= & \text { number of periods to be examined for the } \\
& \text { transient analysis of SMP } \\
P= & \text { matrix of order m of the embedded Markov chain } \\
& \text { in SMP } \\
G^{T}= & \text { square lower-triangular block matrix of order } \\
& \text { T }+1 \text { whose blocks are of order m } \\
Q^{T}= & \text { It represents the kernel of SMP } \\
\Phi^{T}= & \text { block vector of order } \mathrm{T}+1 \text { the block of which are } \\
& \text { square matrices of order m } \\
D^{T}= & \text { block vector of order T+1 the block of which are } \\
& \text { the diagonal square matrix of order } m
\end{aligned}
$$

$V^{T}=$ square lower-triangular block matrix of order $\mathrm{T}+1$ whose blocks are of order $m$

$S^{T}=$ block vector of order $\mathrm{T}+1$ the block of which are the diagonal square matrix of order $m$. The diagonal element of each block t are $s_{i i}=\sum_{j=1}^{m} Q_{i j}(t)$

Given an epoch $\mathrm{T}$ is fixed, matrices $G^{T}$ and $P$, the algorithm solves the linear system (14) for the unknown matrix $\Phi^{T}$ by means of a purely iterative procedure. The algorithm is:

(0) Read the inputs: $m, T, P, G^{T}$

(1) Construct: $Q^{T}, V^{T}, D^{T}$

$$
\begin{aligned}
& V_{(0)}=I ; Q_{(0)}=0 ; S_{(0)}=0 ; D_{(0)}=I \\
& \text { for } t=1 \text { to } T \\
& Q_{(t)}=P^{*} G_{(t)} \\
& \text { for } i=1 \text { to } m \\
& \quad s_{i i}(t)=Q_{i}^{*}(t) \bullet 1 \\
& \text { end for } \\
& \quad V_{(t)}=Q_{(t)}-Q_{t-1} \\
& D_{(t)}=D_{(0)}-S_{(t)} \\
& \text { end for }
\end{aligned}
$$

(2) Given $\Phi_{(0)}=D_{(0)}$, solve for $\Phi^{T}$

$$
\begin{aligned}
& \text { for } t=1 \text { to } T \\
& \Phi_{(t)}=D_{(t)} \\
& \text { for } s=1 \text { to } t \\
& \Phi_{(t)}=\Phi_{(t)}+V_{(s)} \bullet \Phi_{(t-s)} \\
& \text { end for } \\
& \text { end for }
\end{aligned}
$$

(3) Print the results, $\Phi^{T}, Q^{T}$

In the above $(\bullet)$ represent the usual row column matrix product $(*)$ stands for element by element product and (1) is the m-component's sum vector.

In this study, a computer program for solving the evolution equations is developed in the $\mathrm{R}$ statistical software version 2.6.2.

\section{RESULTS AND DISCUSSION}

\subsection{Descriptive Statistics}

The study analyzed data obtained from 1456 AIDS patients during seven years of follow-up. Female represent about $58 \%$ of individuals and $42.5 \%$ of 
transitions concerns patients over 40 years old. The proportion of a patients who is not drug addicted accounted a larger proportion in the sample (about $52.0 \%$ ) compared to those of addicted patients $(42.5 \%)$. The information presented above is summarized in Table 1.

\subsection{Multivariate Analysis}

According to univariate strategies, 10 factors out of 72 possible (6 covariates $* 12$ transitions), were selected. Finally, the multivariate model uses the 8 regression parameters given in Table 2. Women tend to move quickly from state 2 to state 1 . More precisely, Female patients were 1.6 times more likely to move from state 2 to state 1 than those of male patients (adjusted HR = 1.60$, CI $=1.02-2.49)$. Similarly, The hazard of moving from state 3 to state 2 for female patients is about 23\% higher than for male patients (adjusted HR $=1.23$, CI $=1.01-1.48)$. Patients, who is not drug addicted, were 2.49 times more likely to move from state 3 to state 2 than those of drug addicted (adjusted $\mathrm{HR}=2.67, \mathrm{CI}=1.52-4.68)$. Patients with tuberculosis were 2.67 times more likely to move from state 3 to state 4 than those with no tuberculosis (adjusted HR = 2.67, CI $=1.52-4.68)$. Similarly, patients with tuberculosis were 2.28 times more likely to move from state 2 to state 4 than those with no tuberculosis (adjusted HR $=2.28, \mathrm{CI}=1.29-3.99$ ). The hazard of moving from state 3 to state 4 for a patients less than 40 years of old were about $2 \%$ lower than those of a patient greater than 40 years old (adjusted HR $=0.98$, $\mathrm{CI}=0.97-0.99)$. Table 2 reveals this in detail.

\subsection{Results of Semi-Markov for Predicting the Probability of Waiting Time}

Table 3 and Fig. 2 shows that the result of semiMarkov for predicting the probability of waiting time. The conditional probability that a patient stays in state one, two and three for at least 30 months are 0.167 , 0.186 and 0.199 respectively. It is increasing with increasing seriousness of the disease. Within the good states, it is more likely for a patient to stay in a worse state than in a better one. Of course, the death state is an absorbing state, i.e., once a patient enters the death state he/she stays in same state forever. It is also interesting to find out that the conditional probability of staying in same state until a given number of month decreases with increasing time. So there is a possibility of changing from one state to another which is a non-zero probability. Table 3 and Fig. 2 reveal this in detail.

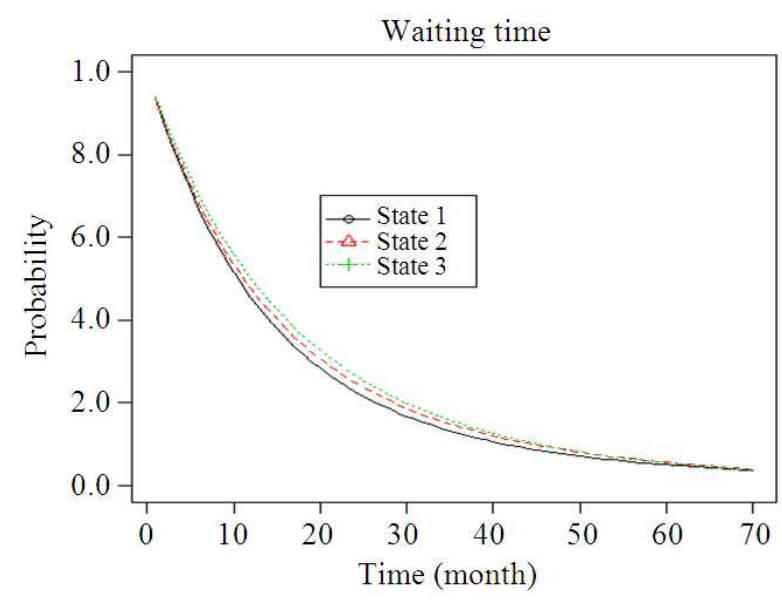

Fig. 2. The probability that a patient stays in same state of disease for at least $t$ months

Table 1. Characteristics of 1456 patients diagnosed with AIDS under Antiretroviral Treatment (ART)

\begin{tabular}{ll}
\hline Variables & Frequency (\%) \\
\hline Sex [n (\%)] & \\
Female & $853(58.6)$ \\
Male & $603(41.4)$ \\
Drug Addicted [n (\%)] & \\
No & $757(52.0)$ \\
Yes & $619(42.5)$ \\
Age [n(\%)] & \\
$<40$ & $922(63.3)$ \\
$\geq 40$ & $534(36.7)$ \\
Baseline Weight [n (\%)] & \\
$<60$ kg & $925(63.5)$ \\
$\geq 60$ kg & $531(36.5)$ \\
TB Status [n (\%)] & \\
Negative & $1085(74.5)$ \\
Positive & $371(25.5)$ \\
Baseline Immunological State [n (\%)] & \\
SI: CD4 $\geq 500$ & $348(23.9)$ \\
SII: $(200<$ CD4<500) & $549(37.7)$ \\
SIII: (CD4 $\leq 350)$ & $559(38.4)$ \\
Transition between States [n (\%)] & \\
$1 \rightarrow 1$ & $152(10.4)$ \\
$1 \rightarrow 2$ & $52(3.6)$ \\
$1 \rightarrow 3$ & $116(8)$ \\
$1 \rightarrow 4$ & $28(1.9)$ \\
$2 \rightarrow 1$ & $206(14.1)$ \\
$2 \rightarrow 2$ & $126(8.7)$ \\
$2 \rightarrow 3$ & $167(11.5)$ \\
$2 \rightarrow 4$ & $50(3.4)$ \\
$3 \rightarrow 1$ & $97(6.7)$ \\
$3 \rightarrow 2$ & $226(15.5)$ \\
$3 \rightarrow 3$ & $157(10.8)$ \\
$3 \rightarrow 4$ & $79(5.4)$ \\
\hline &
\end{tabular}


Table 2. Multiple multi-state model in patients diagnosed with AIDS under ART, Amhara Referral Hospitals, Amhara Region, Ethiopia

\begin{tabular}{llcl}
\hline Variables & Transition & $\begin{array}{l}\text { Adjusted HR } \\
(95 \% \text { CI for its HR) }\end{array}$ & $\begin{array}{c}\text { Adjusted HR } \\
\text { p-value }\end{array}$ \\
\hline Sex & & & $1.60(1.02,2.49)$ \\
Female Vs male* & $2 \rightarrow 1$ & $1.23(1.01,1.48)$ & $0.040^{* *}$ \\
& $3 \rightarrow 2$ & $0.54(0.34,0.85)$ & $0.047^{* *}$ \\
& $3 \rightarrow 4$ & $2.67(1.52,4.68)$ & $0.008^{* *}$ \\
TB status & $3 \rightarrow 4$ & $2.28(1.29,3.99)$ & $0.001^{* *}$ \\
Positive Vs negative* & $2 \rightarrow 4$ & $2.49(1.48,4.19)$ & $0.004^{* *}$ \\
Drug addicted & $3 \rightarrow 2$ & $0.96(0.97,0.98)$ & $0.001^{* *}$ \\
No Vs Yes* & $3 \rightarrow 4$ & $0.98(0.97,0.99)$ & $0.019^{* *}$ \\
Age & $3 \rightarrow 4$ & & $0.000^{* *}$ \\
$\leq 40$ Vs $>40^{*}$ & & &
\end{tabular}

(*) Reference Category; $(* *)$ Hazard Ratio (HR) is a significant at $\alpha=0.05$

Table 3. The probability that a patient stays in same state of disease for at least " $T$ " months

\begin{tabular}{lllllllllllll}
\hline States & $\mathrm{T}=1$ & $\mathrm{~T}=5$ & $\mathrm{~T}=10$ & $\mathrm{~T}=15$ & $\mathrm{~T}=20$ & $\mathrm{~T}=25$ & $\mathrm{~T}=30$ & $\mathrm{~T}=35$ & $\mathrm{~T}=40$ & $\mathrm{~T}=50$ & $\mathrm{~T}=60$ & $\mathrm{~T}=70$ \\
\hline State 1 & 0.933 & 0.712 & 0.515 & 0.378 & 0.283 & 0.216 & 0.167 & 0.132 & 0.105 & 0.071 & 0.050 & 0.036 \\
State 2 & 0.936 & 0.725 & 0.534 & 0.401 & 0.306 & 0.237 & 0.186 & 0.149 & 0.120 & 0.080 & 0.056 & 0.039 \\
State 3 & 0.941 & 0.742 & 0.558 & 0.424 & 0.326 & 0.254 & 0.199 & 0.157 & 0.125 & 0.081 & 0.054 & 0.037 \\
\hline
\end{tabular}

\subsection{Discussion}

The Markov process framework developed by Kay (1986), continued by Gentlemann et al. (1985) and applied by Anderson et al. (1991; Foucher et al., 2005; D'Amico et al., 2009; Giuseppe et al., 2007; Goshu and Dessie, 2013), for studying the effect of disease indicators on survival has been used in this study for constructing survival probabilities for different states of disease progression on HIV/AIDS, introducing nonhomogeneity in time. The present paper is also to estimate transitions between states based on CD4 counts and to predict the probability of waiting time in the starting state until month $t$. The use of the multi-state model, as compared to the Cox regression, improved substantially the understanding of variation in risk factors related to the evolution of this chronic. Foucher et al. (2005) used multi-state models to estimate transitions between states based on VL and CD4 counts.

The most significant difference between studies that use multi-state models is the definition of the different states. Although CD4 count, VL and the incidence of opportunistic infections are used to assess AIDS progression from a clinical point of view, few studies have used these multiple variables. The high cost of the VL assays is a major obstacle to the adoption of this test, therefore restricting its availability compared to CD4 cell counts test. As a result, we found that gender, age, drug addicted and TB status were predictors of the intensity of transitions between different states. This result is similar to the result obtained in previous study (Oliveira et al., 2013; Foucher et al., 2005).

The conditional probability of waiting time in the starting state until month $t$ decreases with increasing of time; while with increasing CD4 count, the probability of waiting time in the starting state until month $t$ decreases. Specifically, AIDS patient who is in the third state of the disease may have a highest waiting time until month $t$ compared to the patients who is in the first and second stage of the disease with a given time. This result is similar to the result obtained in previous studies (Goshu and Dessie, 2013; Giuseppe et al., 2007; Corradi et al., 2004).

\section{CONCLUSION}

The semi-Markov process model is applied to capture the AIDS dynamic progression of a patient. The model considers the randomness of the time that a patient spends in a given state of the disease. The following can be concluded from this study.

When estimating survival in patients with chronic diseases, such as AIDS, that involve the transition between different states, it is fundamentally important to consider progression between the different degrees of chronic. AIDS multi-state modeling is capable of 
identifying the factors associated with each transition between the degrees of chronic defined by CD4 count ranges. The multi-state model analysis showed that the major predictors of the intensity of transitions between different states of HIV/AIDS patients were gender, age, drug addicted and TB status.

Within the good states, it is more likely for a patient to stay in a worse state than in a better one at any time of the process. The death state is an absorbing state, i.e., once a patient is death state he/she stays in same state forever. The conditional probability of staying in same state until a given number of month decreases with increasing time. So there is a possibility of changing from one state to another with non-zero probability.

The dynamic nature of the AIDS progression is confirmed with particular findings that there is more likely to be in worse state than better one unless interventions are made. It is recommendable to keep up the ongoing ART treatment services in most effective ways with the careful considerations of recent disease status of patients.

\section{ACKNOWLEDGEMENT}

My sincere appreciation and thanks go to my staffs of Statistics Department at Bahir Dar University for their unreserved knowledge sharing and cooperation. My appreciation also goes to Felege-Hiwot Referral Hospital, Ethiopia ART members and clinic staff for their cooperation during the time of data collection. Last but not the least; I would like to acknowledge my friend Addisu Abebe (PhD in Economics) for their kind advice and material support.

\section{REFERENCES}

Anderson, K.M., P.M. Odell, P.W. Wilson and W.B. Kannel, 1991. Cardiovascular disease risk profiles. Am. Heart J., 121: 293-8. PMID: 1985385

Barbu, V.S. and N. Limnios, 2009. Semi-Markov Chains and Hidden Semi-Markov Models toward Applications: Their Use in Reliability and DNA Analysis. 1st Edn., Springer Science and Business Media, New York, ISBN-10: 0387731733, pp: 240.

Baryarama, F., J.Y.T. Mugisha and L.S. Luboobi, 2005. An HIV/AIDS model with variable force of infection and its application to the epidemic in Uganda. Am. J. Applied Sci., 2: 1274-1278. DOI: 10.3844/ajassp.2005.1274.1278
Blasi, J. and R. Manca, 2004. Numerical treatment of homogeneous and non-homogeneous semi-Markov reliability models. Commun. Stat. Theory Meth., 33: 697-714. DOI: 10.1081/STA-120028692

Corradi, G., J. Janssen and R. Manca, 2004. Numerical treatment of homogeneous semi-Markov processes in transient case-a straightforward approach. Methodol. Comput. Applied Probability, 6: 233-246. DOI: 10.1023/B:MCAP.0000017715.28371.85

D’Amico, G., J. Janssen and R. Manca, 2005. Homogeneous semi-Markov reliability models for credit risk management. Dec. Econo. Finance, 28: 79-93. DOI: $10.1007 / \mathrm{s} 10203-005-0055-8$

D'Amico, G., J. Janssen, D. Giuseppe and R. Monica, 2009. HIV progression through two different temporal scales according to non-homogeneous semi-Markov models. Proceedings of the 13th International Conference on Applied Stochastic Models and Data Analysis (MDA' 09), pp: 473-476.

Davidov, O. and M. Zelen, 2000. Designing cancer prevention trials: A stochastic model approach. Stat. Med., 2: 1983-1995. DOI: 10.1002/10970258(20000815)19:15<1983::AIDSIM534>3.0.CO;2-E

Foucher, Y., E. Mathieu, P. Saint-Pierre, J. Durand and J. Daures, 2005. A semi-Markov model based on generalized Weibull distribution with an illustration for HIV disease. Biometrical J., 47: 825-833. DOI: 10.1002/bimj.200410170

Gentlemann, R.C., J.F. Lawless, J.C. Lindsey and P. Yan, 1985. Multi-state Markov models for analysing incomplete disease history data with illustrations for HIV disease. Stat. Med., 13: 805-821. DOI: 10.1002/sim.4780130803

Gil, J., A. Alioum, C. Ketzoian, J.C. Desport and M. Druet-Cabanac et al., 2007. Disease progression and survival in ALS: First multi-state model approach. Amyotroph. Lateral Scler., 8: 224-9. DOI: 10.1080/17482960701278562

Giuseppe, D., G. D’Amico, A. Girolamo, J. Janssen and S. Iacobelli et al., 2007. A stochastic model for the HIV/AIDS dynamic evolution. Math. Problems Eng., 2007: 65636-65649. DOI: $10.1155 / 2007 / 65636$

Goshu, A.T. and Z.G. Dessie, 2013. Modelling progression of HIV/AIDS disease stages using semiMarkov processes. J. Data Sci., 11: 269-280. 
Jackson, C., L. Sharples, S. Thompson, S. Duffy and E. Couto, 2003. Multistate Markov models for disease progression with classification error. J. Royal Stat. Society, 52: 193-193. DOI: 10.1111/14679884.00351

Janssen, J. and N. Manca, 2001. Numerical solution of non-homogeneous semi-Markov processes in Transient case. Methodol. Comput. Applied Probability, 3: 271-279. DOI: 10.1023/A: 1013719007075

Kay, R., 1986. A Markov model for analysing cancer markers and disease states in survival studies. Biometrics, 42: 855-865. DOI: 10.2307/2530699

Keiser, O., H. Tweya, P. Braitstein, F. Dabis and P. MacPhail et al., 2010. Mortality after failure of antiretroviral therapy in sub-Saharan Africa. Trop. Med. Int. Health, 15: 251-8. DOI: 10.1111/j.13653156.2009.02445.x

Koethe, J.R., M.I. Limbada, M.J. Giganti, C.K. Nyirenda and L. Mulenga et al., 2010. Early immunologic response and subsequent survival among malnourished adults receiving antiretroviral therapy in Urban Zambia. AIDS, 24: 2117-21. DOI: 10.1097/QAD.0b013e32833b784a

Maciulis, A., A. Paunksnis, V. Barzdziukas, L. Kriauciuniene and D. Buteikiene et al., 2009. Digital model of blood circulation analysis system. Informatica, 20: 539-554.

Masala, G., G. Cannas and M. Micocci, 2014. Survival probabilities for HIV infected patients through semiMarkov processes. Biometrical Lett., 51:13 -36. DOI: $10.2478 /$ bile-2014-0002,

Mathieu, E., Y. Foucher, P. Dellamonica and J.P. Daures, 2007. Parametric and non homogeneous semi-Markov process for HIV control. Methodol. Comput. Applied Probability, 9: 389-397. DOI: 10.1007/s11009-007-9033-7

Muralidhar, S., M. Bala, R.K. Jain, M. Malhotra and K. Ray, 2010. Hepatitis B and C positivity in various categories of human immunodeficiency virus seropositive individuals in a regional STD centre-an eight-year evaluation of trends and risk factors. Am. Med. J., 1: 103-108. DOI: 10.3844/amjsp.2009.103.108
Neuhaus, J., B. Angus, J.D. Kowalska, A. La Rosa and J. Sampson et al., 2010. Risk of all-cause mortality associated with nonfatal AIDS and serious nonAIDS events among adults infected with HIV. AIDS, 24: 697-706. DOI: 10.1097/QAD.0b013e3283365356

Oliveira, R.V.C., S.E. Shimakura, D.P. Campos, F.P. Victoriano and S.R. Ribeiro et al., 2013. Multi-state models for defining degrees of chronicity related to HIV-infected patient therapy adherence. Cad. Saúde Pública, 29: 801-811. PMID: 23568309

Pan, S.L., H.M. Wu, A.M. Yen and T.H. Chen, 2007. A Markov regression random-effects model for remission of functional disability in patients following a first stroke: A Bayesian approach. Stat. Med., 26: 5335-53. PMID: 17676712

Pradeep, M.A., M. Thiruvalluvan and V. Dhamodharan, 2010. A study on erythrocytic determination of anemic condition among human immunodeficiency virus sero positives. Am. Med. J., 1: 80-83. DOI: 10.3844/amjsp.2009.80.83

Pèrez-Ocón, R., J.E. Ruiz-Castro, M.L. Gamiz-Perez, 2001. A piecewise Markov process for analysing survival from breast cancer in different risk groups. Stat. Med., 20: 109-122. PMID: 11135351

Satten, G.A. and M.R. Sternberg, 1999. Fitting semiMarkov models to interval-censored data with unknown initiation times. Biometrics, 55: 507-513. DOI: 10.1111/j.0006-341X.1999.00507.x

Shih, H.C., P. Chou, C.M. Liu and T.H. Tung, 2007. Estimation of progression of multi-state chronic disease using the Markov model and prevalence pool concept. BMC Med. Inform. Dec. Mak., 7: 3434. DOI: $10.1186 / 1472-6947-7-34$

UNAIDS, 2013. Report on the Global HIV/AIDS Epidemic. United Nations Programme on HIV/AIDS, Geneva.

Viladent, C. and A. Van Ackere, 2007. HIV/AIDS modeling, a two-angle-retrospective: Toward a generic deterministic model for pattern II countries? University of Lausanne. 